\title{
Aggregative processes in open systems: simulations and detailed thermodynamic analysis by means of the IdEP-IdLA model
}

\author{
Processus agrégatifs en systèmes ouverts : simulations et analyse \\ thermodynamique détaillée à l'aide du modèle IdEP-IdLA
}

\author{
Vittorio Cocchi ${ }^{1}$, Rossana Morandi ${ }^{2}$ \\ ${ }^{1}$ A.T.I. Rome, Italy, ing.vcocchi@gmail.com \\ ${ }^{2}$ University of Florence, Italy, rossana.morandi@unifi.it
}

\begin{abstract}
The IdEP-IdLA mathematical model, already used in previous works for the study of aggregative processes in closed systems, is here re-proposed for an in-depth study of some thermodynamic aspects of aggregative reactions far from equilibrium. The responses provided by the model when the system reaches the stationary state, are in line with the thermodynamics of non equilibrium: in particular, the numerical results obtained confirm the theorem of minimum entropy production, thus confirming the correctness of the theoretical approach and the reliability of the method. As a result, the model is pushed to simulate, far from equilibrium, the behaviour of dissipative structures: the codified heteropoietic aggregation is acknowledged as the proper mechanism that, within the model, reproduces the thermodynamics of these phenomena in which disorder generates order. The resulting entropic balance initiates a line of reasoning on the causes of dissipative structures.

RÉSUMÉ. Le modèle mathématique IdEP-IdLA, déjà utilisé dans des travaux antérieurs pour l'étude des processus agrégatifs en systèmes fermés, est ici re-proposé pour une étude approfondie de certains aspects thermodynamiques des réactions agrégatives loin de l'équilibre. Les réponses fournies par le modèle lorsque le système atteint l'état stationnaire, sont en accord avec la thermodynamique de non équilibre: en particulier, les résultats numériques obtenus confirment le théorème de production d'entropie minimale, confirmant ainsi la justesse de l'approche théorique et la fiabilité de la méthode. En conséquence, le modèle est poussé à simuler, loin de l'équilibre, le comportement des structures dissipatives: l'agrégation hétéro-poïétique codifiée est reconnue comme le mécanisme propre qui, au sein du modèle, reproduit la thermodynamique de ces phénomènes dans lesquels le désordre génère l'ordre. Le bilan entropique qui en résulte initie un raisonnement sur les causes des structures dissipatives.

KEYWORDS. Entropy, Mathematical modeling, Aggregative processes, Markov sources, Chance and necessity, Open systems, Dissipative structures.

MOTS-CLÉS. Entropie, Modélisation mathématique, Processus agrégatifs, Sources de Markov, Hasard et nécessité, Systèmes ouverts, Structures dissipatives.
\end{abstract}

\section{Foreword}

In previous works [Ref.1 and Ref.2] the IdEP-IdLA model was applied to the study of the aggregative processes of closed heterogeneous ${ }^{1}$ gaseous systems where the ideal gas equation is accepted as valid. It is now proposed to address the case of open systems, in non-equilibrium conditions due to the continuous exchange of energy and matter with the environment. Remembered that ideal elementary particles (IdEP) and related aggregates (IdLA) have a linear frame, free of internal vibrations, the formulas drawn as a result of mentioned works are re-proposed below.

a) The reference exchange reaction is:

$$
\alpha X O^{*}+(1-\alpha) O \leftrightarrow \sum_{\lambda=1}^{\Lambda} v_{O \lambda} O_{\lambda-1}^{*} O+\alpha X
$$

\footnotetext{
${ }^{1}$ In this context, the term heterogeneous indicates a mixture of known composition of homogeneous substances of known quality as well.
} 
where

- $\alpha$ is the molar fraction of the giver compounds

- $X O^{*}$ are the giver compounds consisting of one IdEP $X$, called the grey particle, and one IdEP $O^{*}$ of $\zeta_{O^{*}}$ different colours, called the active primary particle

- $O$ are the free IdEPs, called passive primary particles, of $\zeta_{0}$ different colours

- $O_{\lambda-1}^{*} O$ represents the generic IdLA of length $\lambda$ (with $\Lambda=$ maximum value of $\lambda$ ) variously composed of $\lambda-1$ active primary particles and a single passive primary particle at the end of the sequence

- $v_{O \lambda}$ is the molar fraction of IdLAs of length $\lambda$.

b) The molar fractions of reactants and products as the reaction progresses ( $\xi$, extent of reaction) are:

$v_{X O^{*}}(\xi)=(1-\xi) \alpha$

$v_{O}(\xi)=(1-\xi)(1-\alpha)$

$v_{\lambda}(\xi)=\xi v_{O \lambda}$

$v_{\text {IdLA }}(\xi)=\xi(1-\alpha)$

$v_{X}(\xi)=\xi \alpha$

c) $E_{b}$ is the energy that must be supplied to a mole of giver compounds to break the internal bonds and $E_{f}$ is the energy released by a mole of aggregates as a result of the formation of new bonds, both in [KJoule/mole] $\left(\varepsilon_{j}^{b}\right.$, energy stored in each of the $\zeta_{O^{*}}$ different types of $X-O^{*}$ bonds in the giver compounds; $\varepsilon_{j k}^{f}$, energy stored in each of the $\left(\zeta_{O^{*}}{ }^{2}+\zeta_{O^{*}} \zeta_{O}\right)$ different types of $O^{*}-O^{*}$ and $O^{*}-O$ bonds within the IdLAs; $l_{j}^{b}$, the number of $j$-type bonds between $\zeta_{O^{*}}$ types of $X-O^{*}$ bonds globally present in the initial system; $l_{j k}^{f}$, the number of $j k$-type bonds between the $\left(\zeta_{O^{*}}{ }^{2}+\zeta_{O^{*}} \zeta_{O}\right)$ types of $O^{*}-O^{*}$ and $O^{*}-O$ bonds globally present in the IdLAs produced by a mole of giver compounds):

$\begin{aligned} E_{b} & =\frac{1}{\alpha} \sum_{j=1}^{\zeta_{O^{*}}} l_{j}^{b} \varepsilon_{j}^{b} \\ E_{f} & =\frac{1}{\alpha} \sum_{j=1}^{\zeta_{O^{*}}} \sum_{k=1}^{\left(\zeta_{\left.O^{*}+\zeta_{O}\right)}\right.} l_{j k}^{f} \varepsilon_{j k}^{f}\end{aligned}$

d) $h_{O}$ is the entropy of the descriptor $\left[p_{i}\right]$ of the population of passive primary IdEPs; $h_{X O^{*}}$ is the entropy of the descriptor $\left[p_{j}\right]$ of giver compounds; $h_{X}$ is the entropy of the descriptor of grey particles; $h_{I d L A}$ is the entropy of the IdLA descriptor:

$h_{O}=\sum_{i=1}^{\zeta_{O}} p_{i} \ln \frac{1}{p_{i}}=\gamma_{O} \ln \zeta_{O} \quad\left(\gamma_{O}\right.$, disequilibrium factor of $\left.\left[p_{i}\right]\right)$

$h_{X O^{*}}=\sum_{j=1}^{\zeta_{O^{*}}} p_{j} \ln \frac{1}{p_{j}}=\gamma_{O^{*}} \ln \zeta_{O^{*}} \quad\left(\gamma_{O^{*}}\right.$, disequilibrium factor of $\left.\left[p_{j}\right]\right)$

$h_{X}=0 \quad$ (as grey particles are all of the same colour)

$h_{\text {IdLA }}=(1-\eta)\left[h_{O}+\frac{\alpha}{1-\alpha} h_{X O^{*}}+\frac{1}{1-\alpha} h_{\alpha}\right]$

$\left(\eta\right.$, coding factor ranging from 0 to 1 and $\left.h_{\alpha}=\alpha \ln \frac{1}{\alpha}+(1-\alpha) \ln \frac{1}{1-\alpha}\right)$

e) $\rho$ is the allocation inertia in $\left[\mathrm{m}^{2} \mathrm{Kg}^{5 / 2}\right]$ where $\mu$ and $\delta$ respectively are the mass in $[\mathrm{Kg}]$ and linear dimension in $[\mathrm{m}]$ of the IdEP:

$\rho=\delta^{2} \sqrt{\mu^{5}}$ 
f) $v_{O}$ is the volume of the allocation cell of the primary passive particles; $v_{X O^{*}}$ is the volume of the allocation cell of the giver compounds; $v_{I d L A}$ is the volume of the allocation cell of IdLAs, where $p_{\lambda}$ is the probability that the generic IdLA has length $\lambda$; all of them in $\left[\mathrm{m}^{3}\right]$ :

$v_{O}=v_{X}=v_{I d E P}=\frac{2,8568510^{-112}}{\rho}$

$v_{X O^{*}}=2^{-\frac{9}{2}} v_{I d E P}$

$v_{I d L A}=\prod_{\lambda=1}^{\Lambda}\left(v_{I d E P} \lambda^{-\frac{9}{2}}\right)^{p_{\lambda}}$

$\ln v_{X O^{*}}=\ln v_{I d E P}-\frac{9}{2} \ln 2$

$\ln v_{I d L A}=\ln v_{I d E P}-\frac{9}{2} \Sigma(\alpha)$

(where $\left.\Sigma(\alpha)=\sum_{\lambda=1}^{\Lambda} p_{\lambda} \ln \lambda\right)$

g) $S_{O}, S_{X O^{*}}, S_{X}, S_{I d L A}$ and $S$ are the entropies of the reactants, of the products and of the mixture respectively in [Joule $\left./{ }^{\circ} \mathrm{Kmole}\right]$. All of them, as a function of the extent of reaction $\xi(R=$ $8,314463 \mathrm{Joule} /{ }^{\circ} \mathrm{Kmole}$ is the gas constant; $n_{A}=6,022140910^{23}$ is the number of Avogadro; $T$ is the absolute temperature, in $\left[{ }^{\circ} \mathrm{K}\right] ; V$ is the molar volume in $\left.\left[\mathrm{m}^{3}\right]\right)$ :

$$
\begin{aligned}
& S_{O}(\xi)=\frac{5}{2} R(1-\xi)(1-\alpha) \ln T+R(1-\xi)(1-\alpha)\left[\ln \frac{V}{(1-\xi)(1-\alpha) n_{A}}-\ln \left(v_{O}\right)+1+h_{O}\right] \\
& S_{X O^{*}}(\xi)=\frac{5}{2} R(1-\xi) \alpha \ln T+R(1-\xi) \alpha\left[\ln \frac{V}{(1-\xi) \alpha n_{A}}-\ln \left(v_{X O^{*}}\right)+1+h_{X O^{*}}\right] \\
& S_{X}(\xi)=\frac{5}{2} R \xi \alpha \ln T+R \xi \alpha\left[\ln \frac{V}{\xi \alpha n_{A}}-\ln \left(v_{X}\right)+1+h_{X}\right] \\
& S_{I d L A}(\xi)=\frac{5}{2} R \xi(1-\alpha) \ln T+R \xi(1-\alpha)\left[\ln \frac{V}{\xi(1-\alpha) n_{A}}-\ln \left(v_{I d L A}\right)+1+h_{I d L A}\right] \\
& S(\xi)=S_{O}(\xi)+S_{X O^{*}}(\xi)+S_{X}(\xi)+S_{I d L A}(\xi)
\end{aligned}
$$

h) $\Delta S_{R}$ is the reaction entropy in [Joule $\left./{ }^{\circ} \mathrm{Kmole}\right]\left(\xi_{0}\right.$, extent of reaction at the equilibrium):

$$
\begin{aligned}
& \Delta S_{R}=R\left[\xi_{0} \ln \frac{1}{\xi_{0}}+\left(1-\xi_{0}\right) \ln \frac{1}{\left(1-\xi_{0}\right)}\right]+R \xi_{0}(1-\eta) h_{\alpha}+ \\
& +R \xi_{0}(1-\alpha)\left[\frac{9}{2} \sum_{\lambda=1}^{\Lambda} p_{\lambda} \ln \lambda-\eta \gamma_{O} \ln \zeta_{O}\right]-R \xi_{0} \alpha\left[\frac{9}{2} \ln 2+\eta \gamma_{O^{*}} \ln \zeta_{O^{*}}\right]
\end{aligned}
$$

i) $H_{O}, H_{X O^{*}}, H_{X}, H_{I d L A}$ and $H$ are the enthalpies of the reactants, of the products and of the mixture respectively in [KJoule/mole]. All of them as a function of the extent of reaction $\xi\left(\Delta E=E_{b}-\right.$ $E_{f}$, molar energy balance)

$$
\begin{aligned}
& H_{O}(\xi)=\frac{7}{2}(1-\alpha)(1-\xi) R T \\
& H_{X O^{*}}(\xi)=\frac{7}{2} \alpha(1-\xi) R T+\alpha \xi E_{b} \\
& H_{X}(\xi)=\frac{7}{2} \alpha \xi R T \\
& H_{I d L A}(\xi)=\frac{7}{2} \xi(1-\alpha) R T-\alpha \xi E_{f} \\
& H(\xi)=\frac{7}{2} R T+\alpha \xi \Delta E
\end{aligned}
$$

j) $\Delta H_{R}$ is the reaction enthalpy in [KJoule/mole]:

$$
\Delta H_{R}=H\left(\xi_{0}\right)-H(0)=\alpha \xi_{0} \Delta E
$$

k) Derivatives of entropies in $\left[\mathrm{Joule} /{ }^{\circ} \mathrm{Kmole}\right]$ with respect to the extent of reaction are:

$$
\begin{aligned}
& \frac{d S_{O}}{d \xi}=-R(1-\alpha)\left[\frac{5}{2} \ln T+\ln \frac{V}{(1-\xi)(1-\alpha) n_{A}}-\ln \left(v_{O}\right)+h_{O}\right] \\
& \frac{d S_{X O^{*}}}{d \xi}=-R \alpha\left[\frac{5}{2} \ln T+\ln \frac{V}{(1-\xi) \alpha n_{A}}-\ln \left(v_{X O^{*}}\right)+h_{X O^{*}}\right]
\end{aligned}
$$




$$
\begin{aligned}
& \frac{d S_{X}}{d \xi}=R \alpha\left[\frac{5}{2} \ln T+\ln \frac{V}{\xi \alpha n_{A}}-\ln \left(v_{X}\right)+h_{X}\right] \\
& \frac{d S_{I d L A}}{d \xi}=R(1-\alpha)\left[\frac{5}{2} \ln T+\ln \frac{V}{\xi(1-\alpha) n_{A}}-\ln \left(v_{I d L A}\right)+h_{I d L A}\right] \\
& \frac{d S}{d \xi}=\frac{d S_{O}}{d \xi}+\frac{d S_{X O^{*}}}{d \xi}+\frac{d S_{X}}{d \xi}+\frac{d S_{I d L A}}{d \xi}= \\
& =R\left[\left(\ln \frac{1}{\xi}-\ln \frac{1}{1-\xi}\right)+(1-\alpha)\left(\frac{9}{2} \Sigma(\alpha)-\eta h_{O}\right)-\alpha\left(\frac{9}{2} \ln 2+\eta h_{X O^{*}}\right)+(1-\eta) h_{\alpha}\right]
\end{aligned}
$$

1) $A(\xi)$ is the affinity:

$$
A(\xi)=T \frac{d S}{d \xi}-\alpha \Delta E
$$

m) $\lambda^{*}$ is the weighted average of the IdLAs length, regardless of the aggregation rule:

$$
\lambda^{*}=\frac{1}{1-\alpha}
$$

n) The matching rule between the coding factor $\eta$ e $\Sigma(\alpha)$ is assumed to be

$$
\begin{aligned}
& \eta \rightarrow \Sigma(\alpha)=\Sigma_{\min }(\alpha)+\eta\left[\Sigma_{\max }(\alpha)-\Sigma_{\min }(\alpha)\right] \\
& \text { where: } \quad \Sigma_{\min }(\alpha)=(1-\alpha) \sum_{\lambda=1}^{\Lambda} \alpha^{\lambda-1} \ln \lambda ; \Sigma_{\max }(\alpha)=\ln \frac{1}{1-\alpha}
\end{aligned}
$$

\section{Stationary conditions close to equilibrium}

In closed systems, after a sufficiently long time (depending on the reaction rate) the affinity $A(\xi)$ assumes zero value at an extent of reaction $\xi=\xi_{0} \leq 1$. An open context, on the other hand, implies that the system can exchange not only energy but also matter with the surrounding environment. If the flow of matter is continuous, the affinity can never be zero and therefore the system is not able to achieve the equilibrium. It will rather search for a dynamic balance between the concentrations of reactants and products. If the gradients of the thermodynamic forces supporting the reaction are sufficiently restrained so that a linear relationship exists between these and the matter and energy flows, then it is known that the system tends to reach a steady state of non-equilibrium. We propose to set the material balance and the entropic balance in such condition.

Let us suppose that the place where, at constant pressure and temperature, the transformation of the reactants into products takes place, consists of a portion of space with a constant volume $V$. The reaction is kept active by a constant supply of $\varphi$ moles/sec of reactants as well as by a constant removal of $\varphi$ moles/sec of a mixture of reactants and products, so as to stabilize the concentration of the different species in the volume $V$. We can hypothesize, without compromising the generalities, that the material supply $\varphi$ is represented by a continuous flow of infinitesimal closed volumes where reactants are transformed without material exchange with the outside until each volume is extracted from $V$ (see Fig.1 where volume $V$ is represented as a cylinder with base B and length $\mathrm{L}$ and elementary volumes as cylinders with the same base B and infinitesimal thickness). 


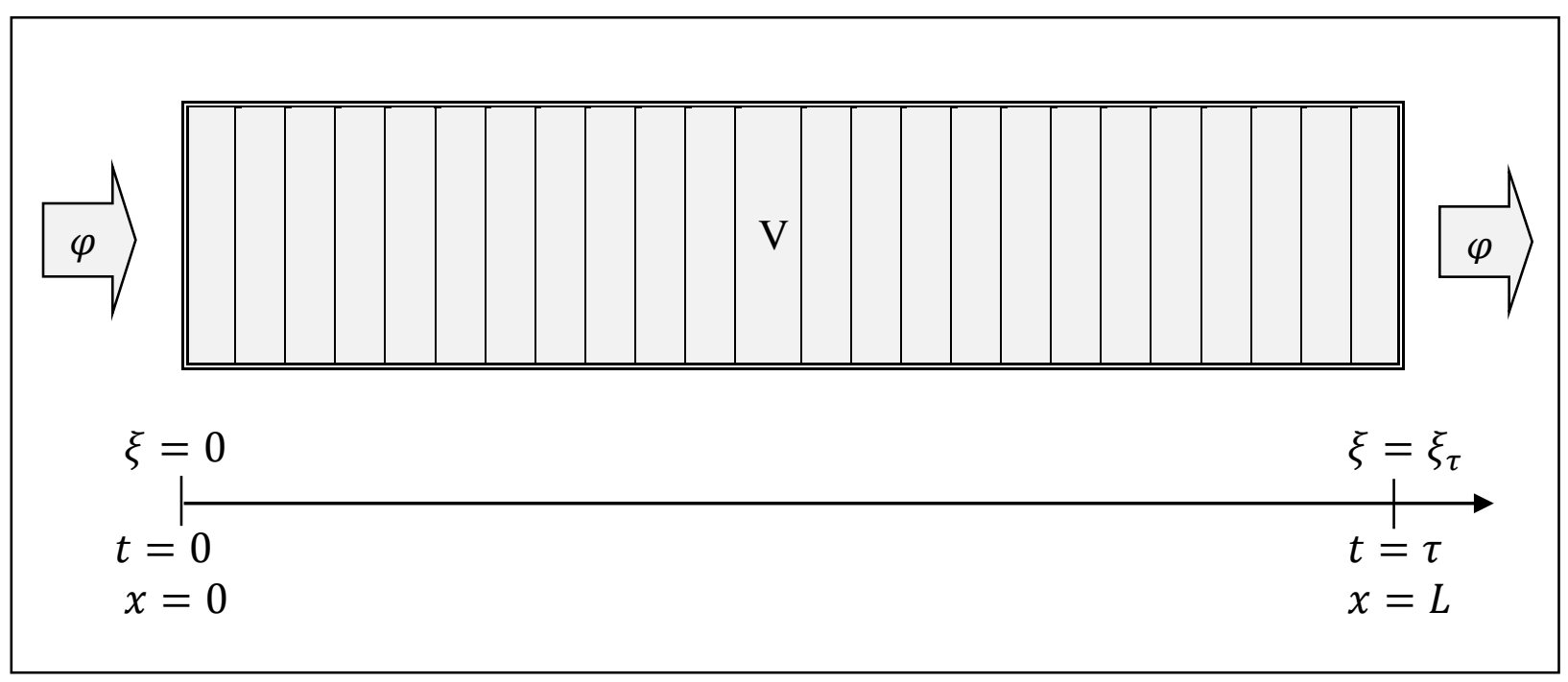

Figure 1. Schematic representation of a system in conditions of stationary non-equilibrium

The (average) time of permanence $\tau$ of infinitesimal volumes inside $V$ is then:

$\tau=\frac{1}{\varphi} \quad[\mathrm{moles} /(\mathrm{moles} / \mathrm{sec})=\mathrm{sec}]$

Consequently, $\xi_{\tau}$ will indicate the extent of reaction characterizing the mixture of reactants and products that is extracted from volume $V$ after the time $\tau$.

It is generally accepted that the development of the process depends on the relationship between time and the concentrations of reactants and products. Then let us suppose that our reaction is a first order one: that means that there is direct proportionality between the reaction velocity and the fraction of the giver compounds $v_{X O^{*}}(\xi)-v_{X O^{*}}\left(\xi_{0}\right)$ supporting the reaction. The reaction velocity $v$ can then be expressed as follows:

$$
v=\frac{d \xi}{d t}=-\frac{1}{\alpha} \frac{d v_{X O^{*}}(t)}{d t}=k\left[v_{X O^{*}}(\xi)-v_{X O^{*}}\left(\xi_{0}\right)\right]<0
$$

where $k$ is the rate constant. Recalling [1/a], [14] takes the form:

$$
\frac{d \xi}{d t}=k \alpha\left(\xi_{0}-\xi\right)
$$

from which:

$$
\frac{1}{\left(\xi_{0}-\xi\right)} d \xi=\alpha k d t \quad d t=\frac{1}{\alpha k\left(\xi_{0}-\xi\right)} d \xi
$$

By integrating between the initial instant $(t=0 \rightarrow \xi=0)$ and the generic time $t$ (and therefore the corresponding extent of reaction $\xi(t)$ ) we obtain the relations

$$
\begin{aligned}
& \xi(t)=\xi_{0}\left(1-e^{-\alpha k t}\right) \\
& t(\xi)=\frac{1}{\alpha k} \ln \frac{\xi_{0}}{\left(\xi_{0}-\xi\right)}
\end{aligned}
$$

Therefore, if the aggregative reaction is of the first order, the extent of reaction $\xi_{\tau}$ characterizing the extracted mixture, due to [16/a], takes the form:

$$
\xi_{\tau}=\xi_{0}\left(1-e^{-\alpha k \tau}\right)
$$


and the concentrations of the different species can immediately be calculated by means of [1/a-e]. Therefore the quality of the mixture continuously extracted from the system, expressed by the extent of reaction $\xi_{\tau}$, depends on what would be the extent of reaction at equilibrium $\xi_{0}$, on the $\alpha$ concentration of the giver compounds and on the aggregated parameter $k \tau$ that takes into account the speed of the aggregative process (by means of $k$ ) and the material flow (by means of $\tau=1 / \varphi$ ). The graphs in Fig.2 show the trends of the ratio $\xi_{\tau} / \xi_{0}$ as a function of $\alpha$ for some discrete values of the product $k \tau$. It is evident that the extent of reaction in stationary conditions is closer to the extent of reaction at equilibrium the higher the rate constant and/or the time of permanence.

It should also be observed that the set material balance is completely general and does not depend on the IdLA formation, in fact no particular hypothesis was made on this.

With regard to the entropic balance, it is current practice to interpret the variation of entropy of an open system with respect to time as the sum of two contributions:

$$
\frac{d S}{d t}=\frac{d_{i} S}{d t}+\frac{d_{e} S}{d t}
$$

where the first one represents the change in entropy per unit of time due to irreversible processes within the system, while the second one represents the change of entropy per unit of time due to exchanges of energy and matter. In stationary conditions the variation of entropy in the system is zero and therefore it must be:

$$
\frac{d_{i} S}{d t}=-\frac{d_{e} S}{d t}
$$

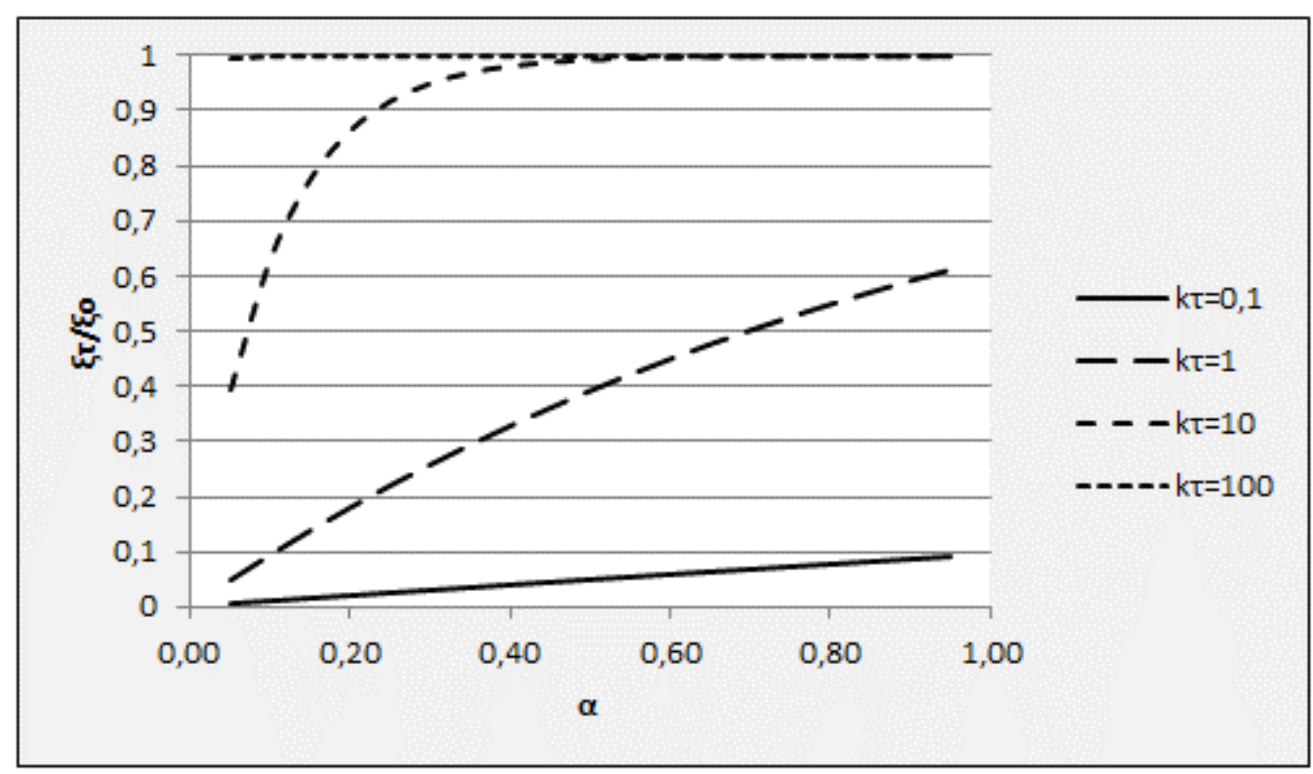

Figure 2. Trend of $\xi_{\tau} / \xi_{0}$ as a function of $\alpha$ for some discrete values of $k \tau$

As for the first term, each of the closed elementary volumes into which we have ideally subdivided the system is the seat of chemical reactions: these, as irreversible processes, provide a positive contribution to the overall increase in entropy. With specific reference to Fig.1, this contribution can be defined by attributing a certain value $s$ of the specific entropy to each position $x$ assumed by the cylindrical elementary volumes within $V .^{2}$ We can then call local entropy

\footnotetext{
${ }^{2}$ The specific entropy thus defined no longer refers to the unit of volume but rather to the unit of length $\left(\mathrm{KJoule} /{ }^{\circ} \mathrm{Km}\right)$ the base $\mathrm{B}$ of the elementary volume being a constant $(d V=B d x)$.
} 
production the quantity $\sigma$ defined as the variation per unit of time of the specific entropy due to internal irreversible processes:

$$
\sigma(x, t)=\frac{d_{i} s(x, t)}{d t}
$$

The change in entropy extended to the whole volume $V$ due to the aggregative reactions is then:

$$
\frac{d_{i} S}{d t}=\int_{V} \sigma d V=\int_{0}^{L} \sigma(x, t) d x=\int_{0}^{L} \frac{d_{i} s(x, t)}{d t} d x=\int_{0}^{L} \frac{a(x)}{T} \frac{d \xi}{d t} d x
$$

where $a(x)$ is the specific affinity pertaining to the generic volume $d V$ in position $x$. At this point, recalling [15], the following is obtained

$$
\frac{d_{i} S}{d t}=\frac{k \alpha}{T} \int_{0}^{L} a(x)\left(\xi_{0}-\xi\right) d x
$$

But as the relationship between $x$ and $\xi$ is bi-univocal, a change of variable can be made:

$$
\frac{d_{i} S}{d t}=\frac{k \alpha}{T} \int_{0}^{\xi_{\tau}} A(\xi)\left(\xi_{0}-\xi\right) \frac{d x}{d \xi} d \xi>0
$$

where $A(\xi)$ is the molar affinity as a function of the extent of reaction and $d x / d \xi$ represents the molar fraction affected by each elementary increment of the extent of reaction.

As for the second term of [19], the change in entropy $d_{e} S / d t$ is due to thermal and material exchanges with the outside:

$$
\frac{d_{e} S}{d t}=\frac{1}{T} \frac{d H}{d t}+\left(\frac{d_{e} S}{d t}\right)_{\text {matter }}
$$

where the quantity $\left(d_{e} S / d t\right)_{\text {matter }}$ represents the exchange of entropy due to the flow of matter. In stationary conditions:

$$
\frac{d_{e} S}{d t}=\frac{1}{T} \frac{G\left(\xi_{\tau}\right)-G(0)}{\tau}<0
$$

where $G(0)-G\left(\xi_{\tau}\right)$ represents the difference in chemical potential between the input flow and the output flow. Replacing then [20] and [21] in [19] we obtain that in stationary conditions it must be

$$
\int_{0}^{\xi_{\tau}} A(\xi)\left(\xi_{0}-\xi\right) \frac{d x}{d \xi} d \xi=-\frac{G\left(\xi_{\tau}\right)-G(0)}{\alpha k \tau}
$$

Note that, contrary to what happens for [20] and [21], in [22] the rate constant $k$ and the material flow $\varphi$ (i.e. the time of permanence $\tau$ ) intervene through the aggregated parameter $k \tau$.

Calculations $^{3}$ for a wide range of the parameters involved demonstrate that the model always produces results that satisfy relation [22]. As an example, the table in Fig.3 shows the results obtained in the case of random autopoietic aggregation for different values of $\alpha$ and of the product

\footnotetext{
${ }^{3}$ The integral in [22] has been solved numerically by discretizing the variation in the extent of reaction. The results obtained by this method are better approximated the finer is the discreteness. In particular the results presented have been obtained with a calculation step $\delta \xi=0,001$. Moreover, with the same discretization the error is more contained the more $\xi_{\tau}$ approaches $\xi_{0}$ therefore for higher values of $\alpha$ and $k \tau$.
} 
$k \tau$, with $\Delta E=-20, h_{O}=h_{X O^{*}}=1,5$ and $\rho=10^{-82}$. The table also shows the value of the affinity of the mixture of reactants and products, calculated ${ }^{4}$ as:

$$
A=\int_{0}^{\xi_{\tau}} A(\xi) d \xi
$$

It is evident that for systems that find their non-equilibrium stationary condition very close to the equilibrium condition $\left(\xi_{\tau}\right.$ very close to $\left.\xi_{0}\right)$, that is when the material flow $\varphi$ is very low and/or the rate constant is very high, the value of $A$ tends to zero. The last column also shows the value of the parameter $A / R T$, which must be $<<1$ in order to have a linear relationship between the thermodynamic forces (the concentration gradients) and the thermodynamic flows (the exchanges of matter and heat): that is the condition for the system to be considered close to equilibrium. The stability of the system is therefore all the more assured the greater the aggregate parameter $k \tau$.

\begin{tabular}{|c|c|c|c|c|c|c|c|c|c|}
\hline \multirow[b]{2}{*}{$\alpha$} & \multirow[b]{2}{*}{$\mathrm{k \tau}$} & \multirow[b]{2}{*}{$\xi_{0}$} & \multirow[b]{2}{*}{$\xi_{\tau}$} & \multirow[b]{2}{*}{$\mathrm{G}(0)$} & \multirow[b]{2}{*}{$G\left(\xi_{\tau}\right)$} & \multicolumn{2}{|c|}{ CHECK OF [22] } & \multirow[b]{2}{*}{ A } & \multirow[b]{2}{*}{$\mathrm{A} / \mathrm{RT}$} \\
\hline & & & & & & $\int_{0}^{\xi t} A(\xi)\left(\xi_{0}-\xi\right) \frac{d x}{d \xi} d \xi$ & $\frac{G\left(\xi_{\tau}\right)-G(0)}{\alpha k \tau}$ & & \\
\hline \multirow{4}{*}{0,1} & 0,1 & \multirow{4}{*}{0,754} & 0,0075 & \multirow{4}{*}{$-58,116$} & $-58,246$ & 12,539 & $-13,000$ & 16,720 & 0,00675 \\
\hline & 1 & & 0,0718 & & $-58,955$ & 8,307 & $-8,390$ & 11,526 & 0,00465 \\
\hline & 10 & & 0,4766 & & $-61,153$ & 3,025 & $-3,037$ & 5,673 & 0,00229 \\
\hline & 100 & & 0,7540 & & $-61,589$ & 0,346 & $-0,347$ & 0,890 & 0,00036 \\
\hline \multirow{4}{*}{0,3} & 0,1 & \multirow{4}{*}{0,948} & 0,0280 & \multirow{4}{*}{$-60,371$} & $-60,888$ & 17,044 & $-17,233$ & 18,243 & 0,00736 \\
\hline & 1 & & 0,2457 & & $-63,517$ & 10,454 & $-10,487$ & 12,577 & 0,00507 \\
\hline & 10 & & 0,9008 & & $-67,642$ & 2,419 & $-2,424$ & 5,470 & 0,00221 \\
\hline & 100 & & 0,9480 & & $-67,686$ & 0,243 & $-0,244$ & 0,601 & 0,00024 \\
\hline \multirow{4}{*}{0,5} & 0,1 & \multirow{4}{*}{0,987} & 0,0481 & \multirow{4}{*}{$-62,121$} & $-63,113$ & 19,703 & $-19,840$ & 20,447 & 0,00825 \\
\hline & 1 & & 0,3884 & & $-67,927$ & 11,585 & $-11,612$ & 14,584 & 0,00588 \\
\hline & 10 & & 0,9803 & & $-72,835$ & 2,140 & $-2,143$ & 5,990 & 0,00242 \\
\hline & 100 & & 0,9870 & & $-72,839$ & 0,214 & $-0,214$ & 0,577 & 0,00023 \\
\hline \multirow{4}{*}{0,7} & 0,1 & \multirow{4}{*}{0,995} & 0,0673 & \multirow{4}{*}{$-63,463$} & $-64,960$ & 21,260 & $-21,386$ & 22,093 & 0,00891 \\
\hline & 1 & & 0,5009 & & $-71,775$ & 11,853 & $-11,874$ & 16,077 & 0,00649 \\
\hline & 10 & & 0,9941 & & $-76,638$ & 1,880 & $-1,882$ & 6,097 & 0,00246 \\
\hline & 100 & & 0,9950 & & $-76,638$ & 0,188 & $-0,188$ & 0,650 & 0,00026 \\
\hline \multirow{4}{*}{0,9} & 0,1 & \multirow{4}{*}{0,996} & 0,0857 & \multirow{4}{*}{$-64,301$} & $-66,220$ & 21,225 & $-21,322$ & 22,241 & 0,00897 \\
\hline & 1 & & 0,5911 & & $-74,210$ & 10,993 & $-11,010$ & 16,085 & 0,00649 \\
\hline & 10 & & 0,9959 & & $-78,237$ & 1,547 & $-1,548$ & 5,312 & 0,00214 \\
\hline & 100 & & 0,9960 & & $-78,237$ & 0,155 & $-0,155$ & 0,757 & 0,00031 \\
\hline
\end{tabular}

Figure 3. Stationary non-equilibrium conditions of a system with random autopoietic aggregation as a funtion of $\alpha$ and $k \tau\left(\Delta E=-20, h_{O}=h_{X O^{*}}=1,5\right.$ and $\left.\rho=10^{-82}\right)$

\section{The principle of minimum entropy production}

If the model works correctly, the composition that the mixture takes on spontaneously as $\xi$ progresses must be such as to minimize $d_{i} S / d t$. In fact, the theorem of minimum entropy production ${ }^{5}$ requires that an open system in which a linear relationship between thermodynamic forces and flows can be assumed as valid, reaches the non-equilibrium stationary state that makes the internal entropy production minimal. It is possible to verify if and to what extent the model

\footnotetext{
${ }^{4}$ This integral has also been calculated numerically with the discretization step $\delta \xi=0,001$.

${ }^{5}$ See [Ref.3], Cap.17.
} 
responds correctly to this condition by artfully unbalancing some phases of the aggregative process, in respect of the macroscopic boundary conditions. With the same input material flow, the most practical way to unbalance the reaction (keeping constant the chemical-physical characteristics of the mixture of reactants and products coming from $V$ ) is to assume that, in a certain portion of $V$, the material flow undergoes an alteration of the aggregative process (acceleration or slowing down) and that, in another portion of $V$, it undergoes an equal and opposite alteration (slowing down or acceleration). Once the static parameters characterizing the system $\left(\alpha, \zeta_{O}, \zeta_{O^{*}}, \gamma_{O}, \gamma_{O^{*}}, \mu, \delta, \Delta E\right)$, the temperature and pressure conditions $(T$ and $p)$, the aggregation mode $(\eta)$ and the dynamic parameters determining the achievement of the stationary non-equilibrium condition $(k$ and $\varphi)$ have been chosen, the stress test in question can be set according to one of the two schemes represented in Fig.4.

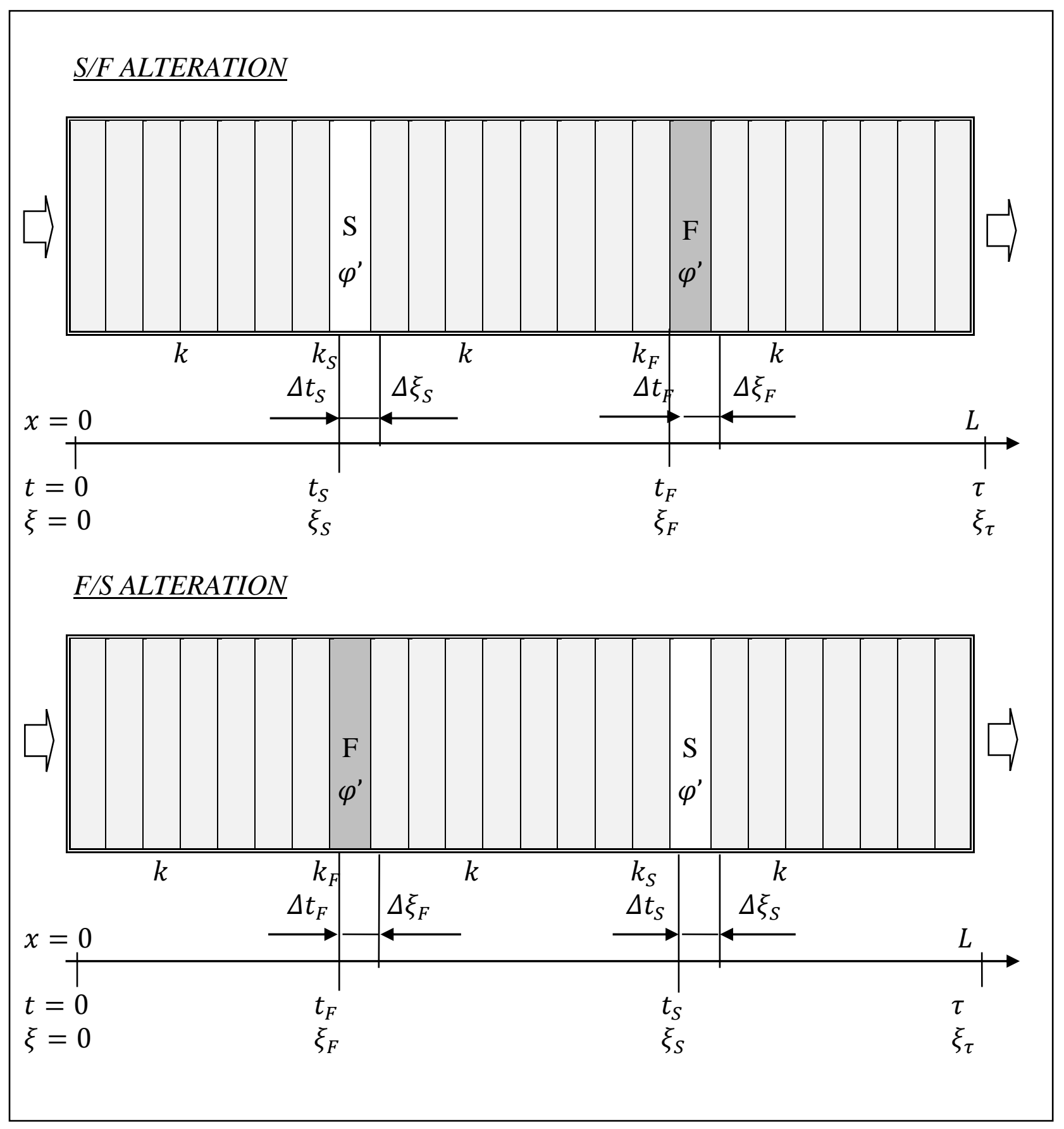

Figure 4. Alterations of steady non-equilibrium conditions 
In it:

- Slow/Fast $(S / F)$ alteration forces an aggregative process characterized by the rate constant $k$, so that the portion $\varphi^{\prime}$ of the material flow $\varphi$ suffers at time $t_{S}$ (i.e. the time that the extent of reaction $\xi_{S}$ is achieved), a slowdown of the reaction: the rate constant decreases from $k$ to $k_{S}$ for a time $\Delta t$, until the extent of reaction is $\xi_{S}+\Delta \xi_{S}$ (necessarily lower than that expected in the undisturbed system at time $t_{S}+\Delta t$ ). Further on, at the time $t_{F}$, characterized by an extent of (perturbed) reaction $\xi_{F}$, an identical fraction $\varphi^{\prime}$ of $\varphi$ undergoes an acceleration of the aggregative process for the same time $\Delta t$, according to a value of the rate constant $k_{F}>k$ such as to bring back the final extent of reaction $\xi_{F}+\Delta \xi_{F}$ to the value expected in the unperturbed system.

- Fast/Slow $(F / S)$ alteration forces an aggregative process in a way conceptually identical to the previous mode but with temporally inverted succession of $k$ alterations.

As they have been defined, both alterations develop and conclude within the system by modifying the internal distribution of the extent of reaction, without affecting at all the chemical-physical characteristics of the material flow. Under perturbed conditions, therefore, the variation of entropy in the unit of time due to thermal and material flows does not change with respect to the stationary regime whereas, according to the abovementioned theorem, the entropy production due to internal irreversible processes must always be greater. Basically, when the open system moves away from the stationary configuration it must be:

$$
\frac{d_{i} S}{d t}>-\frac{d_{e} S}{d t}
$$

Through the IdEP-IdLA model, S/F and F/S alterations have been introduced in a large number of open systems, so disturbing the steady-state, non-equilibrium conditions: inequality [24] has always been confirmed. As an example, the table in Fig.5 shows the results obtained in 12 test cases: 6 for $\mathrm{S} / \mathrm{F}$ alterations and 6 for F/S alterations. The calculations have been carried out assuming the standard conditions for temperature and pressure and assuming, in analogy with the previously examined cases, that:

$-\gamma_{O} \ln \zeta_{O}=\gamma_{O^{*}} \ln \zeta_{O^{*}}=1,5$

- $\rho=10^{-82}$

- $\Delta E=-20 \mathrm{KJ}$ oule/mole

$-\eta=0$.

Values assigned to $\alpha, k$ and $\varphi$ have been chosen so as to have sufficient sensitivity to internal perturbations in terms of increasing entropy production. It must also be remarked that the choice of the portion $\varphi^{\prime}$, of the rate constant perturbations $k_{S} / k$ and $k_{F} / k$ and of $\mathrm{S}$ and $\mathrm{F}$ locations within $V$ are to be considered largely free. However, in order to obtain significant results, the choice has fallen on the perturbation of relatively small fractions of the flow in transit through $V$ and on short but intense alterations of the rate constant. Values of all these parameters are reported in the table of Fig. 5 to define fully the test conditions and, therefore, to make them replicable.

It is evident, from the results, that any perturbation of the stationary state implies an increase in entropy production due to internal irreversible processes. As a consequence, the principle of minimum entropy production always leads the system, whatever the succession of alterations, to react to accidental imbalances by regulating the aggregative process so that an internal order is restored, that respects [19]. 


\begin{tabular}{|c|c|c|c|c|c|c|c|c|}
\hline \multicolumn{3}{|c|}{ S/F ALTERATIONS } & 1 & 2 & 3 & 4 & 5 & 6 \\
\hline \multirow{6}{*}{$\begin{array}{c}\text { Characteristics of } \\
\text { the open system } \\
\text { in the steady } \\
\text { state }\end{array}$} & $\alpha$ & {$[\mathrm{moli} / \mathrm{mole}]$} & 0,5 & 0,5 & 0,5 & 0,1 & 0,5 & 0,5 \\
\hline & $\phi$ & {$[\mathrm{moli} / \mathrm{sec}]$} & 1 & 1 & 1 & 1 & 10 & 10 \\
\hline & $\tau$ & {$[\mathrm{sec}]$} & 1 & 1 & 1 & 1 & 0,1 & 0,1 \\
\hline & $k$ & {$\left[\mathrm{sec}^{-1}\right]$} & 1 & 1 & 1 & 10 & 1 & 1 \\
\hline & $\xi_{0}$ & [moli/mole] & 0,987 & 0,987 & 0,987 & 0,754 & 0,987 & 0,987 \\
\hline & $\xi_{\mathrm{t}}$ & {$[\mathrm{moli} / \mathrm{mole}]$} & 0,388 & 0,388 & 0,388 & 0,477 & 0,048 & 0,048 \\
\hline \multirow{2}{*}{ Alteration } & $\phi^{\prime} / \phi$ & [moli/mole] & \multirow{2}{*}{$4,51 \%$} & \multirow{2}{*}{$2,13 \%$} & \multirow{2}{*}{$4,72 \%$} & \multirow{2}{*}{$4,15 \%$} & \multirow{2}{*}{$4,07 \%$} & \multirow{2}{*}{$20,35 \%$} \\
\hline & $\Delta \mathrm{t} / \tau$ & [sec/sec] & & & & & & \\
\hline \multirow{4}{*}{ S phase } & $t_{p} / \tau$ & {$[\mathrm{sec} / \mathrm{sec}]$} & 0,100 & 0,100 & 0,100 & 0,100 & 0,100 & 0,100 \\
\hline & $\xi_{R}$ & [moli/mole] & 0,100 & 0,048 & 0,048 & 0,072 & 0,005 & 0,005 \\
\hline & $k_{R}$ & {$\left[\mathrm{sec}^{-1}\right]$} & 0,10 & 0,10 & 0,50 & 5,00 & 0,50 & 0,10 \\
\hline & $\Delta \xi_{R}$ & [moli/mole] & 0,002 & 0,001 & 0,011 & 0,014 & 0,001 & 0,001 \\
\hline \multirow{4}{*}{ F phase } & $t_{\mathrm{A}} / \tau$ & {$[\mathrm{sec} / \mathrm{sec}]$} & & 0,299 & 0,299 & 0,210 & 0,200 & 0,370 \\
\hline & $\xi_{A}$ & [moli/mole] & 0,200 & 0,129 & 0,127 & 0,127 & 0,009 & 0,009 \\
\hline & $\mathrm{k}_{\mathrm{A}}$ & {$\left[\mathrm{sec}^{-1}\right]$} & 10 & 10 & 5 & 50 & 4,03 & 2,03 \\
\hline & $\Delta \xi_{A}$ & [moli/mole] & 0,159 & 0,087 & 0,096 & 0,096 & 0,008 & 0,020 \\
\hline \multirow{2}{*}{ Check of [24] } & $-\frac{d_{e} S}{d t}$ & \multirow{2}{*}{ KJoule/ ${ }^{\circ} \mathrm{Ksec}$} & 0,01943 & 0,01943 & 0,01943 & 0,01015 & 0,03304 & 0,03304 \\
\hline & $\frac{d_{i} S}{d t}$ & & $\begin{array}{c}0,02375 \\
22,25 \% \\
\end{array}$ & $\begin{array}{c}0,02148 \\
10,56 \%\end{array}$ & $\begin{array}{c}0,02139 \\
10,10 \% \\
\end{array}$ & $\begin{array}{c}0,01055 \\
3,94 \% 6\end{array}$ & $\begin{array}{c}0,03597 \\
8,8796 \\
\end{array}$ & $\begin{array}{c}0,03379 \\
2,27 \%\end{array}$ \\
\hline \multicolumn{3}{|c|}{ F/S ALTERATIONS } & 7 & 8 & 9 & 10 & 11 & 12 \\
\hline \multirow{6}{*}{$\begin{array}{c}\text { Characteristics of } \\
\text { the open system } \\
\text { in the steady } \\
\text { state }\end{array}$} & $\alpha$ & {$[\mathrm{moli} / \mathrm{mole}]$} & 0,5 & 0,5 & 0,5 & 0,1 & 0,5 & 0,5 \\
\hline & $\phi$ & {$[\mathrm{moli} / \mathrm{sec}]$} & 1 & 1 & 1 & 1 & 10 & 10 \\
\hline & $\tau$ & {$[\mathrm{sec}]$} & 1 & 1 & 1 & 1 & 0,1 & 0,1 \\
\hline & k & {$\left[\mathrm{sec}^{-1}\right]$} & 1 & 1 & 1 & 10 & 1 & 1 \\
\hline & $\xi_{0}$ & [moli/mole] & 0,987 & 0,987 & 0,987 & 0,754 & 0,987 & 0,987 \\
\hline & $\xi_{\tau}$ & [moli/mole] & 0,388 & 0,388 & 0,388 & 0,477 & 0,048 & 0,048 \\
\hline \multirow{2}{*}{ Alteration } & $\phi^{\prime} / \phi$ & [moli/mole] & \multirow{2}{*}{$3,00 \%$} & \multirow{2}{*}{$2,50 \%$} & \multirow{2}{*}{$4,44 \%$} & \multirow{2}{*}{$4,27 \%$} & \multirow{2}{*}{$4,18 \%$} & \multirow{2}{*}{$6,96 \%$} \\
\hline & $\Delta t / \tau$ & {$[\mathrm{sec} / \mathrm{sec}]$} & & & & & & \\
\hline \multirow{4}{*}{ F phase } & $t_{A} / \tau$ & {$[\mathrm{sec} / \mathrm{sec}]$} & 0,100 & 0,051 & 0,051 & 0,100 & 0,100 & 0,100 \\
\hline & $\xi_{A}$ & [moli/mole] & 0,100 & 0,025 & 0,025 & 0,072 & 0,005 & 0,005 \\
\hline & $\mathrm{k}_{\mathrm{A}}$ & {$\left[\mathrm{sec}^{-1}\right]$} & 10,00 & 10,00 & 5,00 & 50,00 & 3,91 & 2,35 \\
\hline & $\Delta \xi_{A}$ & [moli/mole] & 0,138 & 0,113 & 0,101 & 0,131 & 0,008 & 0,008 \\
\hline \multirow{4}{*}{ S phase } & $t_{R} / \tau$ & {$[\mathrm{sec} / \mathrm{sec}]$} & 0,513 & 0,193 & 0,205 & 0,295 & 0,500 & 0,520 \\
\hline & $\xi_{R}$ & [moli/mole] & 0,320 & 0,186 & 0,172 & 0,181 & 0,030 & 0,030 \\
\hline & $k_{R}$ & {$\left[\mathrm{sec}^{-1}\right]$} & 0,1 & 0,1 & 0,5 & 5 & 0,5 & 0,3 \\
\hline & $\Delta \xi_{R}$ & [moli/mole] & 0,001 & 0,001 & 0,009 & 0,010 & 0,001 & 0,001 \\
\hline \multirow{2}{*}{ Check of [24] } & $-\frac{d_{e} S}{d t}$ & \multirow{2}{*}{ KJoule/ ${ }^{\circ} \mathrm{Ksec}$} & 0,01943 & 0,01943 & 0,01943 & 0,01015 & 0,03304 & 0,03304 \\
\hline & $\frac{d_{i} S}{d t}$ & & $\begin{array}{c}0,02231 \\
14,83 \%\end{array}$ & $\begin{array}{c}0,02182 \\
12,31 \% 6\end{array}$ & $\begin{array}{c}0,02127 \\
9,4896\end{array}$ & $\begin{array}{c}0,01056 \\
4,0496\end{array}$ & $\begin{array}{c}0,03590 \\
8,66 \% 6\end{array}$ & $\begin{array}{c}0,03432 \\
3,8796\end{array}$ \\
\hline
\end{tabular}

Figure 5. Principle of minimum entropy production: verification tests

\section{Instability far from equilibrium}

It is well known that, far from equilibrium, the thermodynamic behavior of a system can be very different from the forecasts of minimum entropy production theorem. Beyond certain limits, as a result of random fluctuations due to imperfections or inhomogeneity, the system can lose its stability and find a new stationary configuration in often highly specialized states. It is also well known that towards such new configurations, with a high organization content, systems can converge even 
starting from very different initial conditions. According to modern thermodynamics it is the same dissipative nature of real physical phenomena that causes phase orbits, even if moving from different points, to converge towards a given state (or set of states), decreasing their entropy. These systems are usually referred to as dissipative structures, ${ }^{6}$ introduced by Ilya Prigogine at the end of the 1960s. Random fluctuations and the consequent fall of a highly disordered and unstable system into a more organized state of stationary equilibrium constitute the special dynamic path through which a local order spontaneously arises at the expense of a greater external disorder; a local order to the occurrence of which the statistical thermodynamics of equilibrium would attribute a practically nil probability.

The question therefore arises whether and to what extent the IdEP-IdLA model is able to describe, far from equilibrium, the transition from a stationary state at a low level of organization to a more orderly one. In order to give an answer, the model can be used in two successive phases: it is assumed that the production of aggregates is controlled first by a low value of the coding factor and then by a significant improvement of it. This means that the dissipative jump occurring between the two phases is obtained by working on the memory of the IdLA sequencer whose entropy depends on $\eta$. Finally, it is admitted that the simulation of the phenomenon (at constant temperature and pressure) can be carried out by adopting the following strategy, once the initial system has been fully defined.

A) Achievement of the equilibrium state as a consequence of an autopoietic aggregative process with a low coding factor $\eta_{1}$. This low-coded equilibrium state (LCE state in Fig.6) is representative of a generic complex of possible high entropy starting conditions. If the molar energy balance $\Delta E_{1}$ is taken to be associated with the aggregative reaction, the calculation of the extent of reaction $\xi_{0}^{1}$ at the equilibrium and of the thermodynamic potentials $\Delta H_{R}^{1}, \Delta S_{R}^{1}, \Delta G_{R}^{1}$ is then possible.

B) Moving the system away from the equilibrium state, through the introduction of kinetic parameters $\varphi$ and $k_{1}$. The system then reaches a non-equilibrium stationary state (LCS state in Fig.6). Calculation of the extent of reaction $\xi_{\tau}^{1}$ of the mixture coming from $V$ and calculation of the entropy production $\left(d_{i} S / d t\right)_{1}$ due to internal irreversible processes are then possible.

C) Transition to a higher degree of order (dissipative jump) by imposing a new coding factor $\eta_{2}>\eta_{1}$. It is assumed that such high-coded steady state (HCS state in Fig.6) can be calculated starting from the equivalent in-equilibrium system (HCE state in Fig.6). If the molar energy balance $\Delta E_{2}$ is supposed to be associated to this aggregative reaction, it is easy to obtain the extent of reaction at equilibrium $\xi_{0}^{2}$ and the thermodynamic potentials $\Delta H_{R}^{2}, \Delta S_{R}^{2}, \Delta G_{R}^{2}$. Once the rate constant $k_{2}$ of the new aggregative process is defined, with the flow $\varphi$ remaining unmodified, the new values of $\xi_{\tau}^{2}$ e di $\left(d_{i} S / d t\right)_{2}$ can thus be obtained.

We call retro-equivalence postulate the hypothesis introduced in point $\mathrm{C}$ ). According to this, the calculation in the HCS state can be carried out by assuming as starting point the HCE state, typical of a system identical to that in LCE state but characterized by different values of the coding factor $\left(\eta_{2}>\eta_{1}\right)$ and of the molar energy balance $\left(\Delta E_{2} \neq \Delta E_{1}\right)$. It is also assumed that the same material flow $\varphi$ used to reach LCS state starting from LCE state, is also adopted to move from HCE state to HCS state even if a different value of the rate constant $\left(k_{2} \neq k_{1}\right)$ is to be applied. Basically, we postulate the equivalence of the paths A and B of Fig.6.

\footnotetext{
${ }^{6}$ See [Ref.3], Cap.19.
} 


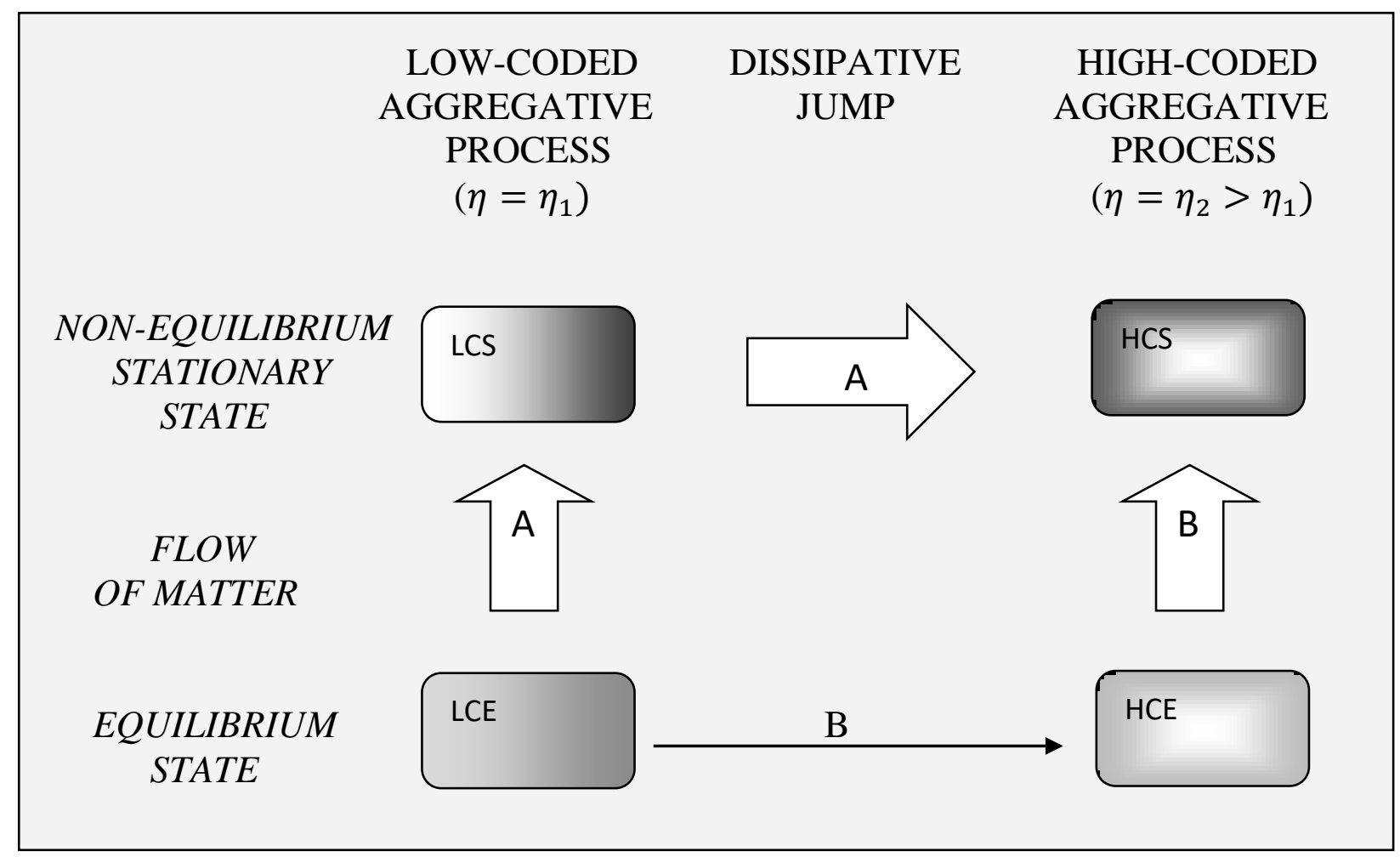

Figure 6. Transition between contiguous states

It should be noted that the proposed mechanism, including the retro-equivalence postulate, has a purely descriptive value, in fact nothing on the causes of the dissipative jump has been said or can be deduced from the pattern. Even with this limitation, the proposed strategy appears acceptable as mathematically manageable within the IdEP-IdLA model, as well as adequate to physical intuition. The question which arises is whether or not it leads to plausible conclusions. The answer lies in the very nature of the phenomenon. Even if marked out by a more ordered complex of aggregates (assured by the fact that $\eta_{2}>\eta_{1}$ ) the system should also be characterized by a higher entropy production, that is the following inequality should be valid

$$
\left(d_{i} S / d t\right)_{2}>\left(d_{i} S / d t\right)_{1}
$$

Since the adopted procedure leaves the values of $\Delta E_{2}$ (in comparison with $\Delta E_{1}$ ), $k_{2}$ (in comparison with $k_{1}$ ) and $\varphi$ substantially unspecified, it is appropriate to examine the effects that these parameters have on entropy production. In the consequent calculations the following parameters have been therefore fixed:

$-\gamma_{O} \ln \zeta_{O}=\gamma_{O^{*}} \ln \zeta_{O^{*}}=1,5$

- $\rho=10^{-82}$

- $\eta_{1}=0\left(\ln v_{\text {ALId }}=\max \right)$

- $\eta_{2}=1\left(\ln v_{A L I d}=\min \right)$.

As far as coding factors are concerned, it is therefore assumed that the dissipative jump takes place starting from a random aggregation toward a forced one, thus creating the maximum increase of order possible. The other parameters necessary to determine the LCE and LCS states have therefore been defined by identifying a reference value and a range of variation for each one as follows:

- concentration of giver compounds:

reference value: $\alpha=0,5$

range of variation $[0,1 / 0,9]$

- molar energy balance before dissipative jump: reference value: $\Delta E_{1}=-20 \mathrm{KJ}$ oule $/ \mathrm{mole}$ 
range of variation $[-10 /-30]$

- material flow:

reference value: $\varphi=0,1$ moles $/ \mathrm{sec}(\rightarrow \tau=10 \mathrm{sec})$

range of variation: $[0,1 / 0,2]$

- rate constant before dissipative jump:

reference value: $k_{1}=0,1 \sec ^{-1}\left(\rightarrow k_{1} \tau=1\right)$

range of variation: $[0,05 / 0,10]$.

Finally, conditions related to the HCS state have been studied by varying the rate constant $k_{2}$ (in particular the ratio $k_{2} / k_{1}$ is used as a parameter) and the molar energy balance $\Delta E_{2}$. The graphs in Fig. 7 show the values of entropy productions $\left(d_{i} S / d t\right)_{1}$ and $\left(d_{i} S / d t\right)_{2}$ (respectively in LCS and HCS states) as a function of $k_{2} / k_{1}$ and $\alpha$ when $\Delta E_{1}, \varphi$ and $k_{1}$ assume the above indicated reference values and $\Delta E_{2}=\Delta E_{1}$. It is evident that:

- when $k_{2} / k_{1}=1$ inequality [25] is never respected and therefore the dissipative jump cannot take place

- as the ratio $k_{2} / k_{1}$ increases, the gap between the values of PCS and HCS entropy production decreases until parity is reached at a rate constant equal to $k_{2}^{*}$. When $k_{2} / k_{1} \geq k_{2}^{*} / k_{1}$ the dissipative jump can take place under the defined conditions (i.e. $\Delta E_{2}=\Delta E_{1}$ )

- for very small $(<0,1)$ or very large $(>0,9)$ values of $\alpha$ there is no reaction velocity able to satisfy inequality [25]

- the lowest values of $k_{2}^{*} / k_{1}$ are obtained for concentrations of giver compounds ranging between 0,5 and 0,7 . In this interval the dissipative jump seems therefore to be facilitated.

Let us now examine what effect the variation of $\Delta E_{2}$ has. In this respect we must consider that a higher coding factor on the occasion of the dissipative jump $\left(\eta_{2}>\eta_{1}\right)$ necessarily implies that the natural attitude to the autopoietic coupling dictated by the matrix of the binding energies is forced; but this also implies that the aggregative process takes place at a more consistent distance from the condition of maximum energy dissipation. It must therefore be:

$$
\Delta E_{2}>\Delta E_{1} \quad\left(\left|\Delta E_{2}\right|<\left|\Delta E_{1}\right|\right)
$$

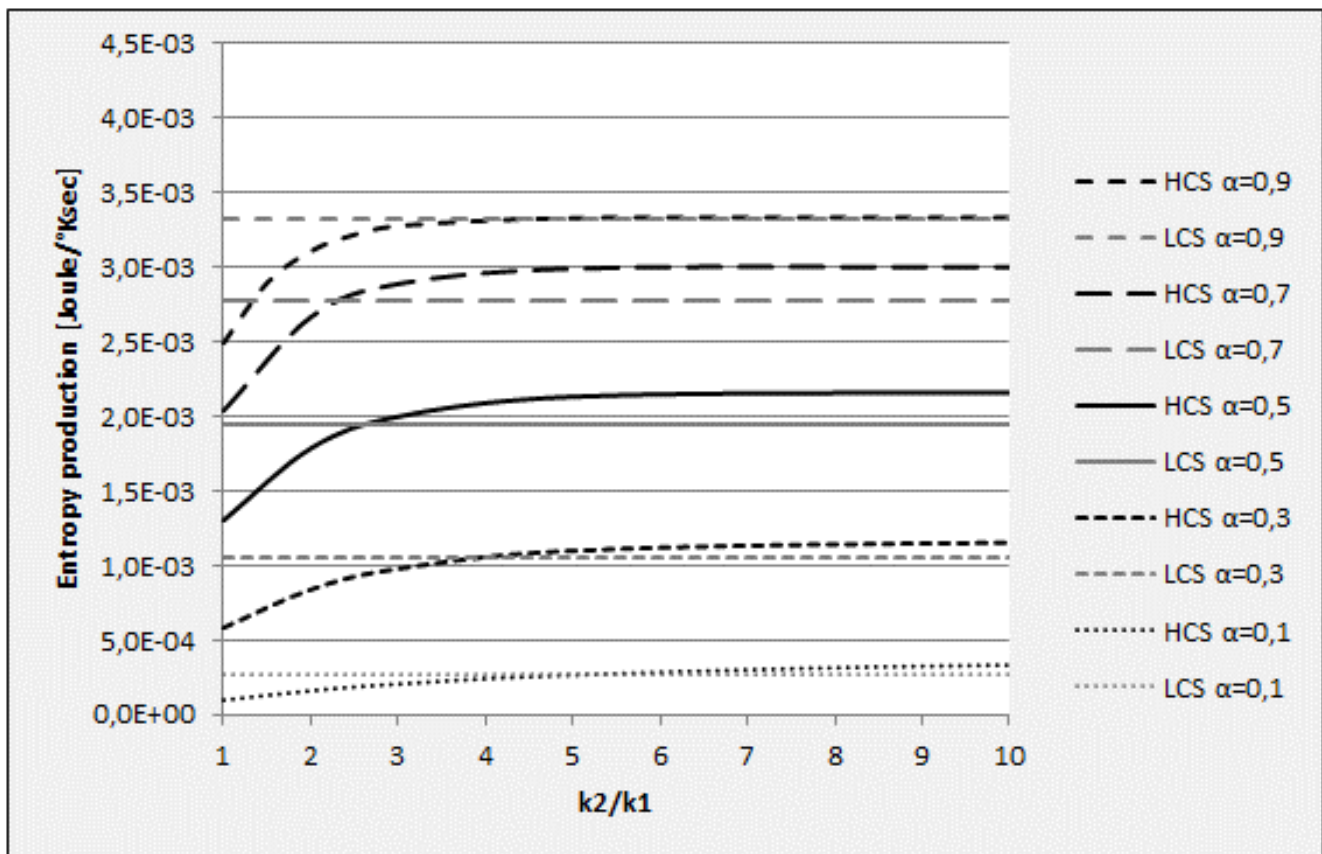

Figure 7. Entropy production in LCS and HCS states as a function of $k_{2} / k_{1}$ and $\alpha$ with $\Delta E_{2}=\Delta E_{1}=-20 \mathrm{KJoule} / \mathrm{mole}$ 
The graphs in Fig.8 show the values of entropy productions $\left(d_{i} S / d t\right)_{1}$ and $\left(d_{i} S / d t\right)_{2}$ as a function of $k_{2} / k_{1}$ and $\Delta E_{2}$ when $\Delta E_{1}, \varphi$ and $k_{1}$ assume the reference values and $\alpha=0,5$. As we can see:

- the decrease in energy dispersion connected with the dissipative jump makes it much more difficult to achieve the condition expressed by [25]

- as $\Delta E_{2}$ becomes less negative, the reaction velocity must increase very much to compensate the effect on $\left(d_{i} S / d t\right)_{2}$. If with $\Delta E_{2}=\Delta E_{1}=-20$ relation [25] is fulfilled when $k_{2} / k_{1}>2,5$, with $\Delta E_{2}=-19$, the value of $k_{2} / k_{1}$ must increase to at least 3,9 and with $\Delta E_{2}=-18$ there is no rate of reaction able to balance the loss of entropy production.

The last remark is clearly due to the fact that a lower molar energy balance has the consequence, all other conditions being equal, of reducing the extent of reaction at equilibrium: when an exothermic aggregative reaction is supported by lower values of dissipated thermal energy, the dynamic equilibrium between reactants and products is reached earlier and the reaction stops, that is $\xi_{0}^{2}<\xi_{0}^{1}$. But in non-equilibrium stationary conditions, keeping the dynamic parameters ( $\varphi$ and also $k$, as we want to proceed by separation of the effects) unmodified, a lower extent of reaction $\xi_{0}$ at the equilibrium will necessarily be matched by a lower extent of reaction $\xi_{\tau}$ of the mixture coming from $V$, as imposed by [17] that is, $\xi_{\tau}^{2}<\xi_{\tau}^{1}$. Consequently, as a mixture less rich in aggregates comes from volume $V$, the entropy production is lowered. This justifies that [26] brings a negative contribution to the occurrence of [25].

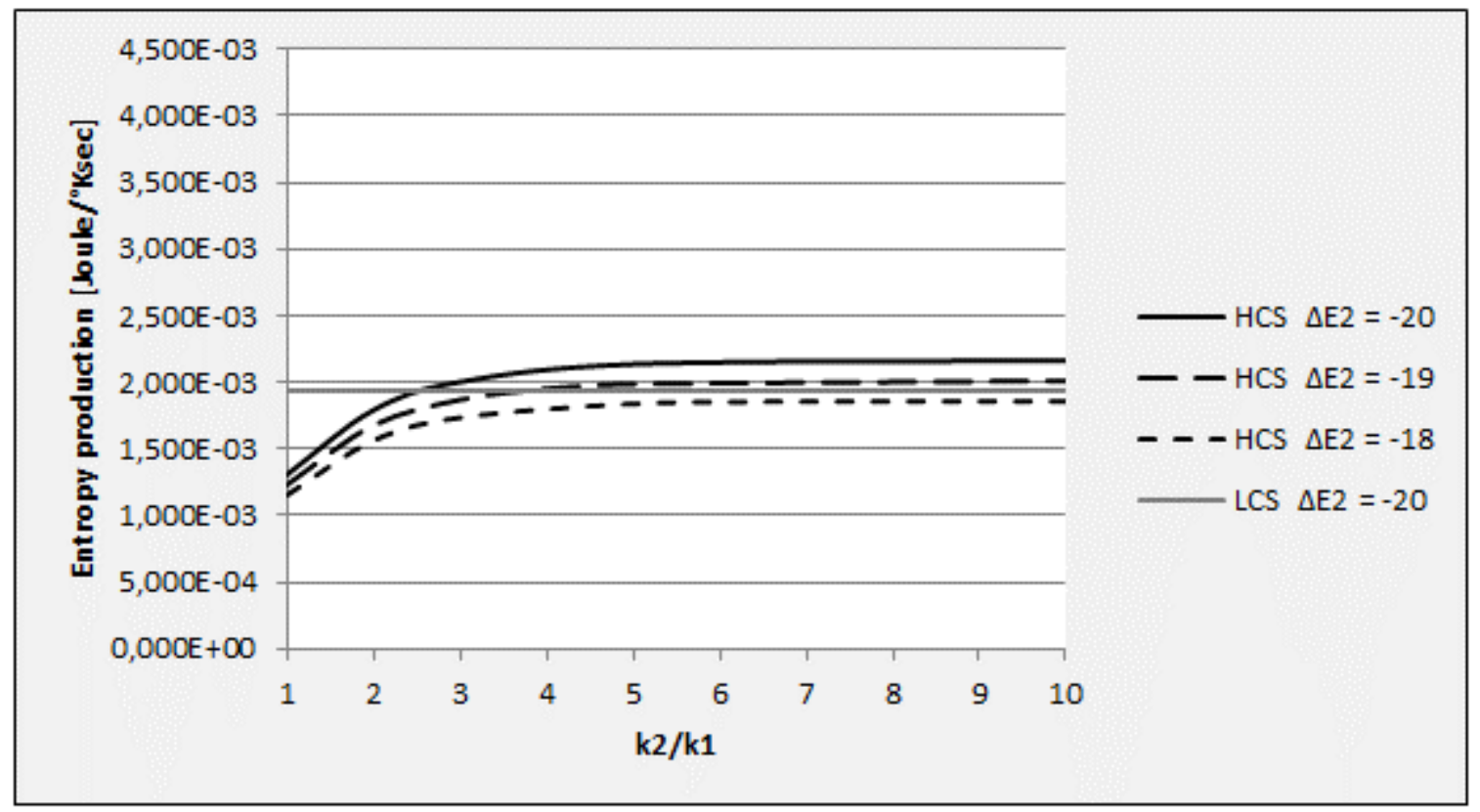

Figure 8. Entropy production in HCS and LCS states as a function of $k_{2} / k_{1}$ and $\Delta E_{2}$ with $\alpha=0,5, \varphi=0,1$ and $k_{1}=0,1$ 


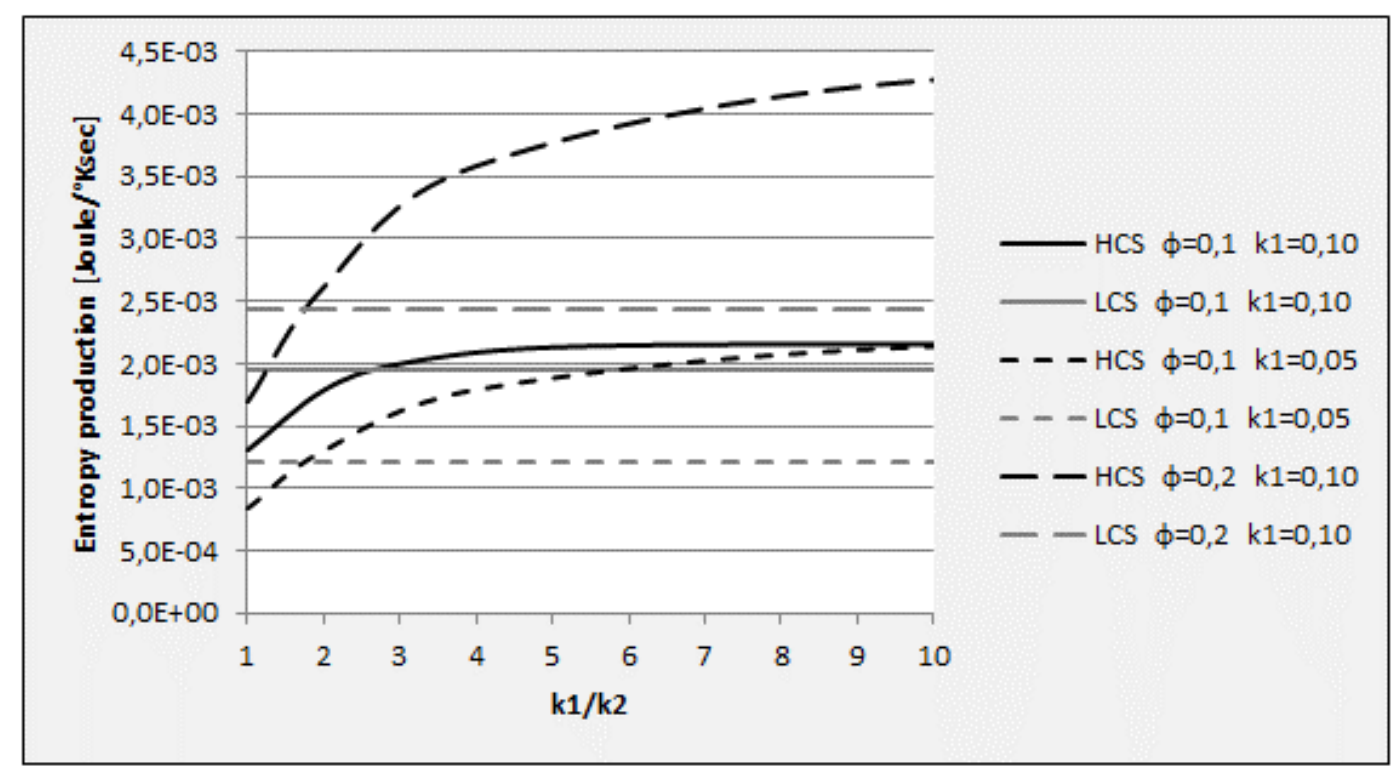

Figure 9. Entropy production in LCS and HCS states as a function of $k_{2} / k_{1}$ when $\varphi$ and $k_{1}$ vary and $\alpha=0,5, \Delta E_{2}=\Delta E_{1}=-20$

It remains to be examined which contributions to the occurrence of [25] are made by the variations of the other reference parameters: $\varphi, k_{1}$ and $\Delta E_{1}$. The graphs in Fig.9 show the values of entropy production as a function of $k_{2} / k_{1}$ when $\varphi$ and $k_{1}$ vary while $\Delta E_{2}=\Delta E_{1}=-20$ and $\alpha=0,5$. As we can see, with respect to the reference condition:

- the increase (the doubling, in our case) of the material flow $\varphi$ lowers the value of $k_{2}^{*} / k_{1}$, making the dissipative jump easier (the decrease would have an opposite effect)

- also the decrease of the rate constant $k_{1}$ before the dissipative jump, reduces $k_{2}^{*} / k_{1}$ ratio. On the contrary, a higher reaction velocity before the jump would increase $k_{2}^{*} / k_{1}$, removing the possibility of the LCS $\rightarrow$ HCS transition.

Finally, the graphs of Fig.10 show the trend of entropy production as a function of $k_{2} / k_{1}$ and $\Delta E_{1}$ when $\varphi=0,1, k_{1}=0,1, \Delta E_{2}=\Delta E_{1}=-20$ and $\alpha=0,5$. It is clear that the more exothermic the aggregative reaction, the lower the value of $k_{2}^{*} / k_{1}$. On the other hand, for low-energy processes there is no reaction velocity capable of fulfilling [25].

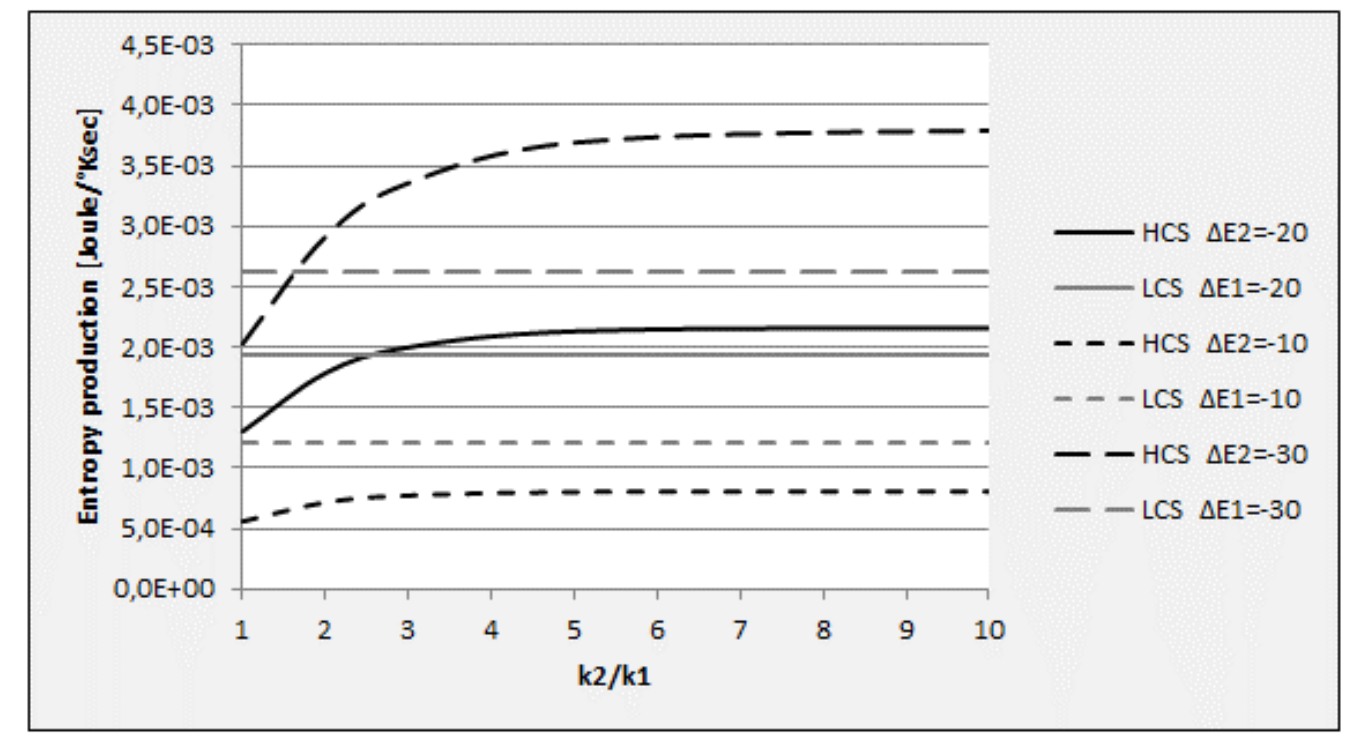

Figure 10. Entropy production in LCS and HCS states as a function of $k_{2} / k_{1}$ and $\Delta E_{1}$ with $\varphi=0,1, k_{1}=$ $0,1, \alpha=0,5$ and $\Delta E_{2}=\Delta E_{1}$ 
In the light of the obtained results, the model indicates that necessary conditions for the formation of stable dissipative structures are the following:

a) the aggregative reaction must be decidedly exothermic ( $\Delta E_{1}$ greater than a critical threshold)

b) the energy balance $\Delta E_{2}$ after the dissipative jump must be only slightly lower than the energy balance $\Delta E_{1}$ before the jump

c) the rate of reaction must increase $\left(k_{2}>k_{1}\right)$. In any case, all other conditions remaining unmodified, such increase may be all the more contained the lower the initial speed constant $k_{1}$ is and the higher the material flow $\varphi$.

The following typical scenario is therefore outlined. A closed system consisting of a balanced mixture of reactants (i.e. $\alpha$ not too far from 0,5) gives rise to a complete, or almost complete, autopoietic aggregative reaction (PCE). The system is gradually moved away from the equilibrium by a gradually increasing material flow $\varphi$ and as a consequence, the system is subject to a sequence of stationary states in which the extent of reaction $\xi_{\tau}$ is gradually lower and lower (depending on the rate constant $k_{1}$ ) while the entropy production increases step by step (see Fig.3). The system gradually becomes less and less stable (see ratio $A / R T$, still in Fig.3). When the PCS state is reached, the system becomes definitively unstable, the coding factor passes from $\eta_{1}$ to $\eta_{2}$, the molar energy balance from $\Delta E_{1}$ to $\Delta E_{2}$ (slightly lower), the rate constant from $k_{1}$ to $k_{2}$ and the system jumps to a new steady state (HCS) characterized by a more orderly aggregate complex and a higher production of entropy, in compliance with [25].

This is what the model describes. But it also suggests that in the context of an aggregative reaction:

- a dissipative jump occurs all the more easily the more similar the binding energies are to each other. In the case of perfect identity among binding energies we have $\Delta E_{2}=\Delta E_{1}$ that is a random aggregation in the LCE state. This justifies, a posteriori, having set $\eta_{1}=0$ and having developed most of the calculations assuming a constant molar energy balance

- if the aggregation in the LCE and LCS states is autopoietic, as the matrix of bonds is unique and unchangeable, aggregation in the HCS state can only be heteropoietic. It is then necessary to assume that ordering agents external to the system become operative only on the occasion of the dissipative jump

- the active presence of ordering agents gives rise to two further considerations of a qualitative nature. First, the ordering agents should also have a catalytic function as this would make the aggregative process not only more orderly but also faster. Secondly, since the ordering agents must necessarily be the site of energy degradation phenomena, the dissipative jump is facilitated by this. The greater the energy dispersed by these entities, the smaller the increase in reaction velocity or material flow required for the system to access the LCS $\rightarrow$ HCS transition.

Whether and to what extent the model is representative of real physical situations depends on the results that can be obtained by imposing realistic values on the different parameters. And this is the subject of a future discussion.

\section{Conclusions}

The IdEP-IdLA model shows a good aptitude for representing the thermodynamics of aggregative reactions in open systems. In particular, it is suitable to describe effectively the ways in which order can be created from the disorder and it determines, also in quantitative terms, the necessary conditions for the emergence of dissipative structures. Since these are considered of particular concern in the biological field, the model presents as a promising tool in the study of aggregations of biochemical interest. 


\section{References}

[Ref.1] V. Cocchi, R. Morandi - Mathematical modeling for the simulation of aggregative processes, Entropie, ISTE Open Science. DOI: 10.21494/ISTE.OP.2021.0667, 2021

[Ref.2] V. Cocchi, R. Morandi - Use of the IdEP-IdLA model for the study of aggregative processes in closed systems, Entropie, ISTE Open Science. 10.21494/ISTE.OP.2021.0741

[Ref.3] D. Kondepudi, I. Prigogine - Modern Thermodynamics, New York, John Whiley \& Sons, 1999 